\title{
Dynamical breaking of shift symmetry in supergravity-based inflation
}

\author{
Anupam Mazumdar, ${ }^{1, *}$ Toshifumi Noumi, ${ }^{2, \dagger}$ and Masahide Yamaguchi ${ }^{3, *}$ \\ ${ }^{1}$ Consortium for Fundamental Physics, Physics Department, Lancaster University, \\ Bailrigg, Lancaster LA1 4YB, United Kingdom \\ ${ }^{2}$ Mathematical Physics Laboratory, RIKEN Nishina Center, Saitama 351-0198, Japan \\ ${ }^{3}$ Department of Physics, Tokyo Institute of Technology, Tokyo 152-8551, Japan
}

(Received 27 May 2014; published 18 August 2014)

\begin{abstract}
Shift symmetry is essential to protect the flatness of the potential, even beyond the super-Planckian vacuum expectation value for an inflaton field. The breaking of the shift symmetry can yield potentials suitable for super-Planckian excursion of the inflaton. The aim of this paper is to illustrate that it is indeed possible to break the shift symmetry dynamically within four-dimensional supergravity prior to a long phase of inflation. Thanks to the shift symmetry, the leading contribution to the inflaton potential is free from the dangerous exponential factor even after its breaking, which is the main obstacle to realizing the super-Planckian inflation in supergravity. But, in our simple model, the resulting inflaton potential is a cosine-type potential rather than the power-law one, and it is difficult to realize a super-Planckian breaking scale, unfortunately.
\end{abstract}

DOI: 10.1103/PhysRevD.90.043519

PACS numbers: $98.80 . \mathrm{Cq}$

\section{INTRODUCTION}

The observations of the cosmic microwave background (CMB) temperature anisotropies [1,2] now strongly support the occurrence of primordial inflation [3] in the early Universe. The observed temperature anisotropy can be well fitted by the primordial perturbations generated during inflation, and the anticorrelation of the temperature $(T)$ and $E$-mode polarization at a large angular scale suggests that the primordial perturbations have been stretched on superhorizon scales $[1,2]$. In addition, very recently, BICEP2 reported the detection of the primordial tensor perturbations through the $B$-mode polarization as [4]

$$
r=0.20_{-0.05}^{+0.07}(68 \% \text { C.L. }),
$$

where $r$ is the tensor-scalar ratio. To explain this large tensor-to-scalar ratio is challenging for cosmology and particle physics because of the Lyth bound [5]; one would expect a super-Planckian excursion of the inflaton field in order to generate large $r$. Of course, the current data can also be explained by the sub-Planckian excursion of the inflaton field [6,7], or via assisted inflation [8] with many copies of the inflaton field [9], where the field displacement $\Delta \phi \simeq 0.1 M_{p} \leq M_{p}$, where $\phi$ is the inflaton and $M_{p} \simeq$ $2.44 \times 10^{18} \mathrm{GeV}$, but here in this paper we are interested in studying the opposite limit, when $\Delta \phi>M_{p}$.

Generally speaking, the super-Planckian excursion of the inflaton is problematic from the effective field theory (EFT) point of view of particle physics and string theory [10].

\footnotetext{
*a.mazumdar@lancaster.ac.uk toshifumi.noumi@riken.jp

¥gucci@phys.titech.ac.jp
}

In particular, within string theory there are many scales, the string scale $M_{s}$, the compactification scale $M_{c}$, and the derived four-dimensional Planck scale, with a spectrum $M_{s} \leq M_{c} \leq M_{p}$. Beyond $M_{s}$, there are quantum corrections not only to the inflaton potential but also to the inflaton kinetic term which can lead to various complications; see [11]. One would require a full nonperturbative completion of gravity, which we lack sorely within string theory as well. Even if we assume that we have only one fundamental scale, such as $M_{p}$, there are many issues pertaining to the validity of an EFT when the field's vacuum expectation value (VEV) goes beyond $M_{p}$. In principle, a gauge singlet inflaton can couple to many degrees of freedom, including the Standard Model and the hidden sector degrees of freedom; see [12]. Typically, the individual inflaton's coupling to matter has to be smaller than $10^{-3}$ to maintain the flatness of the inflaton potential and also to match the density perturbations created during inflation. Of course, there is no fundamental justification to make such couplings smaller other than matching the current constraints arising from the CMB.

Furthermore, there are higher derivative corrections to the inflaton kinetic term (see [11]); if we do not take all infinite higher derivative terms into consideration, there are potential problems with ghosts and quantum instability during inflation. One cannot ignore the higher derivative terms, because a priori one does not know what should be the inflaton's kinetic energy; i.e., the inflaton need not be slow rolling throughout the phase of inflation [11].

In spite of all these challenges, we wish to ask the question of whether we can explain at least such small inflaton couplings to matter and the large inflaton's VEV during inflation within an EFT approach by invoking some symmetry such as shift symmetry. Within EFT, one has to 
ensure that the inflaton's and all other field's kinetic terms are small, and here we simply assume so in some patch of the Universe just to be within the EFT regime [13], though this still relies on anthropic arguments.

In principle, one could imagine a shift symmetry as a fundamental symmetry of nature, which would forbid masses and couplings to an inflaton field. Such a shift symmetry has been for the first time introduced in the context of chaotic inflation in supergravity (SUGRA) $[14,15]$. However, by the same token, if shift symmetry remains unbroken, inflation would never occur in our patch of the Universe. The shift symmetry has to be broken, but in such a way that the breaking remains soft, which could be understood via some dynamics of the fields. A hard breaking can be introduced $[14,15]$, but the predictions can be lost or one has to resort to some anthropic arguments.

The purpose of this paper is to illustrate a concrete model of dynamical shift symmetry breaking. Our model is described within a four-dimensional $\mathcal{N}=1$ SUGRA setup, and the effective inflaton potential results in cosine-type potential [16] without the dangerous exponential factor. Unfortunately, in our simple model, it is difficult to realize the super-Planckian breaking scale like natural inflation. ${ }^{1}$

The organization of the paper is as follows. In the next section, we will introduce shift symmetry; then we will construct a simple scenario of dynamically breaking the shift symmetry in SUGRA and explain how it works. In the final section, we will give our conclusions and discussions.

\section{A BRIEF DISCUSSION ON SHIFT SYMMETRY}

Let us explain how the shift symmetry allows the superPlanckian variation of the inflaton field. Note that this argument is not confined to a supersymmetric (SUSY) theory but applies to a non-SUSY theory. A shift symmetry is characterized by a symmetry under the following transformation of a (real) inflaton field $\phi$ :

$$
\phi \rightarrow \phi+c \quad(c: \text { real constant }) .
$$

As long as this symmetry is exact, the potential of the inflaton is completely flat, and any field variation even beyond the reduced Planck scale $M_{p}$ is allowed. This is an essential idea. However, inflation must end to reheat our Universe; then the shift symmetry must be broken to generate the gradient of the potential.

As far as we know, in all of the models considered so far, the shift symmetry is broken simply by hand or by introducing an auxiliary field, a spurion field, with no kinetic term, whose nonzero VEV is given by hand. For example, in SUGRA models, it is often assumed that the Kähler potential respects the shift symmetry while the superpotential breaks the shift symmetry. In such a case,

\footnotetext{
${ }^{1}$ See Refs. $[17,18]$ for recent works on natural inflation. Also see e.g., Refs. [12,19] for other inflation models in supergravity.
}

any kind of superpotential can appear, because there is no founding principle behind the breaking of shift symmetry. The introduction of a spurion field might cure such ambiguity, because the original action before giving a nonzero VEV to the spurion field respects the shift symmetry in this approach. Then, the interactions, or the forms of the Kähler potential and the superpotential, can be constrained. For example, let us introduce a spurion field $S$ and extend the shift symmetry as [15]

$$
\begin{gathered}
\phi \rightarrow \phi+c \quad(c: \text { real constant }), \\
S \rightarrow S \frac{\phi}{\phi+c} .
\end{gathered}
$$

Then, the combination $S \phi$ is invariant under the shift symmetry. Once this spurion field $S$ takes a nonzero VEV, i.e., $\langle S\rangle=m$, the shift symmetry is broken and the potential is generated. The key points are the following.

(i) The inflaton field $\phi$ always appears in the combination: $\langle S\rangle \phi=m \phi$, where $m \ll M_{p}$. As long as $m \phi \leq M_{p}^{2}$, the EFT treatment is still justified, in spite of the fact that the cutoff scale of the inflaton is now raised to $M_{p}^{2} / m$.

(ii) No super-Planckian masses of fermions and bosons appear, because any interactions of the inflaton including Yukawa and four-point interactions are suppressed by the small scale $m \ll M_{p}$.

(iii) If we take the $m \rightarrow 0$ limit, the shift symmetry is restored. In this sense, this model is technically natural. Thus, chaotic inflation can be naturally realized in this setup, and the model given in Refs. $[14,15]$ is a concrete realization in the context of SUGRA.

However, even in this setup, the nonzero VEV of the field $S$, i.e., the breaking of the shift symmetry, has been introduced by hand, unfortunately, by assuming that it is a spurion field. Needless to say, it is better to break the shift symmetry dynamically, because, otherwise, we cannot control the whole dynamics of the system or evaluate the effects of the shift symmetry breaking adequately. In this paper, we address this issue and propose a concrete model of the dynamical breaking of the shift symmetry in SUGRA.

\section{DYNAMICAL BREAKING OF SHIFT SYMMETRY}

In this section, we are going to construct a concrete model of dynamical breaking of the shift symmetry in $\mathcal{N}=1$ SUGRA. The key observation is that the following superpotential:

$$
W=e^{a \Phi}
$$

is invariant (up to a constant phase) under the shift symmetry, 


$$
\Phi \rightarrow \Phi+i \frac{C}{a}
$$

where $a$ and $C$ are real constants. In fact, the scalar potential in the global SUSY limit is given by

$$
V(\Phi)=a^{2} e^{a\left(\Phi+\Phi^{*}\right)}
$$

which depends only on the real part of $\Phi$. Thus, the shift symmetry on the imaginary part of $\Phi$ remains. ${ }^{2}$ On the other hand, the following superpotential:

$$
W=e^{a \Phi}+e^{-a \Phi}
$$

is not invariant under the shift symmetry. In fact, the scalar potential in the global SUSY limit is given by

$$
V(\phi, \chi)=a^{2}\left[e^{\sqrt{2} a \chi}+e^{-\sqrt{2} a \chi}-2 \cos (\sqrt{2} a \phi)\right]
$$

where

$$
\Phi=\frac{1}{\sqrt{2}}(\chi+i \phi) .
$$

It should be noticed that the scalar potential depends not only on the real part of $\Phi$, i.e., $\chi$, but also on the imaginary part of $\Phi$, i.e., $\phi$. In order to recover the shift symmetry for the second type of the superpotential [see Eq. (8)], we need to introduce a pair of superfields, $S$ and $S$, and another superfield $X$. $^{3}$ Let us now consider the following superpotential:

$$
W_{I}=v\left(S^{n} e^{a \Phi}+\tilde{S}^{n} e^{-a \Phi}\right) X
$$

where $v \ll 1$ is a constant (in Planck units) and $n$ is a positive integer number. This superpotential is invariant under the following shift symmetry:

$$
\begin{aligned}
& \Phi \rightarrow \Phi+i \frac{n C}{a}, \\
& S \rightarrow S e^{-i C}, \\
& \tilde{S} \rightarrow \tilde{S} e^{i C}, \\
& X \rightarrow X .
\end{aligned}
$$

However, in order to realize inflation, this shift symmetry must be broken. For this purpose, we introduce another superfield $T$ and add the following superpotential:

\footnotetext{
${ }^{2}$ From here onwards, we denote the scalar components of the superfields by the same symbols as the corresponding superfields.

${ }^{3}$ The superfield $X$ is necessary not only for recovery of the shift symmetry. It is also useful to guarantee the positivity of the potential during inflation $[14,15,20]$.
}

$$
W_{B}=\lambda T\left(S \tilde{S}-\mu^{2}\right),
$$

where $\lambda \leq \mathcal{O}(1)$ and $\mu \leq \mathcal{O}(1)$ are constants. Then, the total superpotential, which is given by

$$
W=W_{I}+W_{B},
$$

is invariant under the shift symmetry [Eq. (12)], along with

$$
T \rightarrow T
$$

One can easily understand that, once the scalar components of the superfields $\langle S\rangle \neq 0$ and $\langle\tilde{S}\rangle \neq 0$ acquire nonzero VEVs, the shift symmetry is broken dynamically. Let us consider the following Kähler potential of the type ${ }^{4}$ :

$$
K=\frac{1}{2}\left(\Phi+\Phi^{*}\right)^{2}+|S|^{2}+|\tilde{S}|^{2}+|T|^{2}+|X|^{2},
$$

which is invariant under the shift symmetry [Eqs. (12) and (15)] and generates the canonical kinetic terms for all of the fields. Note that shift symmetry will also allow higher order terms, such as $\left(\Phi+\Phi^{*}\right)^{4},\left(\Phi+\Phi^{*}\right)^{6}$, $\ldots,|S|^{4},|S|^{6}, \ldots,|\bar{S}|^{4},|\bar{S}|^{6}, \ldots,|T|^{4},|T|^{6}, \ldots,|X|^{4},|X|^{6} \ldots$, $S^{n} e^{a \Phi}, \tilde{S}^{n} e^{-a \Phi}, \ldots$, etc., where ... contain higher order terms to all infinite orders. These terms will give corrections to the canonical kinetic terms. But, as long as $\left(\Phi+\Phi^{*}\right),|S|,|\tilde{S}|, \ldots \ll 1$, which can be realized dynamically in our model, these corrections are negligible. Of course, at the initial period, we assume the presence of at least one patch of the Universe in which the kinetic energies of all of the fields are smaller than the Planck energy density and subdominant.

The higher derivative terms like $\mathcal{D}_{a} \Phi \mathcal{D}^{a} \Phi^{*}$ in the Kähler potential are also allowed from our symmetry; see [21]. Unless these higher order terms are suppressed by $\left(\Phi+\Phi^{*}\right)^{2}$, for example, the derivative expansion may not be justified because of the super-Planckian value of $\phi$ (see again Ref. [11]). One would need to take all infinite higher derivative corrections in order to avoid ghosts and instability of the vacuum [11]. This would require a complete ultraviolet completion of the inflaton and gravitational sector, which we do not aim to address in this paper. Instead, we make an assumption that the inflationary patch is always within an EFT regime.

Further note that the present model possesses $U(1)_{\mathrm{R}}$ symmetry, under which

\footnotetext{
${ }^{4}$ The linear term of $\Phi+\Phi^{*}$ can appear in the Kähler potential because of the absence of the $Z_{2}$ symmetry. Such an effect causes two effects. The first one is an additional contribution to the $D$ term. The second one is a slight deviation of the minimum of the $\chi$ field during inflation from the global minimum. This deviation is still compatible with the $D$-flat condition, because its deviation exactly cancels out the additional contribution to the $D$ term. So, the essential dynamics remains unchanged and we omit it for simplicity.
} 


$$
\begin{aligned}
\Phi(\theta) & \rightarrow \Phi\left(\theta e^{i \alpha}\right), \\
S(\theta) & \rightarrow S\left(\theta e^{i \alpha}\right), \\
\tilde{S}(\theta) & \rightarrow \tilde{S}\left(\theta e^{i \alpha}\right), \\
X(\theta) & \rightarrow e^{-2 i \alpha} X\left(\theta e^{i \alpha}\right), \\
T(\theta) & \rightarrow e^{-2 i \alpha} T\left(\theta e^{i \alpha}\right) .
\end{aligned}
$$

The scalar potential in $\mathcal{N}=1$ SUGRA is given by

$$
\begin{aligned}
V= & e^{K}\left[\left|v a\left(S^{n} e^{a \Phi}-\tilde{S}^{n} e^{-a \Phi}\right) X+\left(\Phi+\Phi^{*}\right) W\right|^{2}\right. \\
& +\left|n v S^{n-1} e^{a \Phi} X+\lambda T \tilde{S}+S^{*} W\right|^{2}+\mid n v \tilde{S}^{n-1} e^{-a \Phi} X \\
& +\lambda T S+\left.\tilde{S}^{*} W\right|^{2}+\left|\lambda\left(S \tilde{S}-\mu^{2}\right)+T^{*} W\right|^{2} \\
& \left.+\left|v\left(S^{n} e^{a \Phi}+\tilde{S}^{n} e^{-a \Phi}\right)+X^{*} W\right|^{2}-3|W|^{2}\right]+V_{D} .
\end{aligned}
$$

From here onwards, we set $M_{p}=1$. In the above potential, $V_{D}$ represents the $D$-term contribution, which is given by

$$
V_{D}=\frac{e^{2}}{2}\left(|\tilde{S}|^{2}-|S|^{2}+\frac{n}{a}\left(\Phi+\Phi^{*}\right)\right)^{2},
$$

with $e$ being a gauge coupling constant. Such a term can be present if the shift symmetry is gauged by changing the constant parameter $C$ to a spacetime-dependent one $C(x)$.

\section{INFLATIONARY POTENTIAL}

Now, let us take a closer look at the dynamics of this system. First, let us assume that the energy scale of the shift symmetry breaking sector, i.e., $W_{B}$, is much higher than that of the inflation sector, i.e., $W_{I}$, which requires

$$
\lambda^{2} \mu^{4} \gg v^{2} \mu^{2 n} \Leftrightarrow \lambda \gg v \mu^{n-2} .
$$

Under this assumption, the potential energy is roughly given by $V \simeq \lambda^{2} \mu^{4}$ at the onset of inflation and the Hubble expansion rate: $H^{2} \simeq V / 3 \simeq \lambda^{2} \mu^{4} / 3$. At such higher energies, hybrid-type inflation [22,23] can occur, where $T$ cannot take a value larger than unity (in Planck units) due to the exponential factor $e^{K}$ in the potential; see Eq. (18). Then, the mass squared of the field $X$ is estimated to be

$$
m_{X}^{2} \simeq \lambda^{2} \mu^{4}\left(1+|T|^{2}\right) \simeq 3 H^{2}\left(1+|T|^{2}\right),
$$

which dynamically drives the field $X$ to the zero VEV. It can be easily confirmed that, even after this inflation, $m_{X}^{2}$ is always positive, so that $X$ stays at the origin forever. By inserting $X=0$ to the scalar potential, Eq. (18) yields

$$
\begin{aligned}
\left.V\right|_{X=0}= & e^{K}\left[\lambda^{2}|T|^{2}\left(\Phi+\Phi^{*}\right)^{2}\left|S \tilde{S}-\mu^{2}\right|^{2}+\lambda^{2}|T|^{2}\left(\left|\tilde{S}\left(1+|S|^{2}\right)-\mu^{2} S^{*}\right|^{2}+\left|S\left(1+|\tilde{S}|^{2}\right)-\mu^{2} \tilde{S}^{*}\right|^{2}\right)\right. \\
& \left.+\lambda^{2}\left(1-|T|^{2}+|T|^{4}\right)\left|S \tilde{S}-\mu^{2}\right|^{2}+v^{2}\left|S^{n} e^{a \Phi}+\tilde{S}^{n} e^{-a \Phi}\right|^{2}\right]+\frac{e^{2}}{2}\left(|\tilde{S}|^{2}-|S|^{2}+\frac{n}{a}\left(\Phi+\Phi^{*}\right)\right)^{2},
\end{aligned}
$$

and the mass terms for $S$ and $\tilde{S}$ are estimated as

$$
\begin{aligned}
m_{S, \bar{S}}^{2} \simeq & -\lambda^{2} \mu^{2}\left(S \tilde{S}+S^{*} \tilde{S}^{*}\right)+\lambda^{2}|T|^{2}\left(1+\mu^{4}\right)\left(|S|^{2}+|\tilde{S}|^{2}\right) \\
= & \lambda^{2}\left[\left(1+\mu^{4}\right)|T|^{2}+\mu^{2}\right]|\Psi|^{2}+\lambda^{2}\left[\left(1+\mu^{4}\right)|T|^{2}\right. \\
& \left.-\mu^{2}\right]|\bar{\Psi}|^{2}
\end{aligned}
$$

where we have defined

$$
\Psi=\frac{1}{\sqrt{2}}\left(S-\tilde{S}^{*}\right), \quad \bar{\Psi}=\frac{1}{\sqrt{2}}\left(S+\tilde{S}^{*}\right),
$$

and we have taken $n \geq 2$ in Eq. (11). Since $m_{\Psi}^{2} \gg$ $\lambda^{2} \mu^{4} \simeq 3 H^{2}$, the $\Psi$ field has a Hubble-induced mass and quickly settles down to the zero VEV within one Hubble time or so, which implies $S=\tilde{S}^{*}$ and $|S|=|\tilde{S}|$. This condition is compatible with the $D$-term flatness condition $V_{D}=0$, along with $\Phi+\Phi^{*}=0$, which holds true for almost all periods. At this point, we can discuss the dynamics of the fields for two particular scenarios.

(i) $|T| \gtrsim T_{c}$, dynamically preserving shift symmetry.As long as the VEV of $T$ is such that $|T| \gtrsim T_{c} \simeq \mu$, or $m_{\Psi}^{2} \gg \lambda^{2} \mu^{4} \simeq 3 H^{2}$, which also leads dynamically to
$\bar{\Psi}=0$. Therefore, for $|T| \gtrsim T_{c}, S$ and $\tilde{S}$ stay at the origin and the potential $V$ is dominated by $\lambda^{2} \mu^{4}$, leading to the hybrid inflation $[22,23]$.

The SUGRA effects and the one-loop potential coming from the SUSY breaking effects could drive the inflaton field $T$ like in the case of standard hybrid inflation. It should be noticed that, during this inflation, the effective mass squared of the real part of $\Phi, \chi$, is approximately $3 H^{2}$. Therefore, $\chi\left(=\left(\Phi+\Phi^{*}\right) / \sqrt{2}\right)$ quickly rolls down to its minimum, that is, the zero as well. On the other hand, the imaginary part of $\Phi, \phi$, is still arbitrary. That is, the shift symmetry is preserved at this stage.

(ii) $|T| \lesssim T_{c}$, dynamically breaking shift symmetry.-In this case the effective mass squared $m_{\bar{\Psi}}^{2}<0$, with its magnitude is larger than the Hubble parameter squared, the $\bar{\Psi}$ field becomes unstable so that the fields $S$ and $\tilde{S}$ quickly roll down to the minimum of the potential with $S \tilde{S}=\mu^{2}$ and $|S|=|\tilde{S}|$ together with $\Phi+\Phi^{*}=0$, which can be parametrized as

$$
S=\mu e^{i \beta}, \quad \tilde{S}=\mu e^{-i \beta}
$$


with $\beta$ being a real constant. Thus, the fields $S$ and $\tilde{S}$ acquire the nonzero VEVs, which dynamically breaks the shift symmetry.

Further note that, for $S \tilde{S} \simeq \mu^{2}$, the effective mass squared of $T, m_{T}^{2}$, is estimated as

$$
m_{T}^{2} \simeq 2 \lambda^{2} \mu^{2},
$$

which mainly comes from the second and third terms in the right-hand side of the first line in Eq. (22). Thus, after the end of hybrid inflation, $T$ quickly settles down to its minimum, i.e., $\langle T\rangle=0$. Then, the effective scalar potential, with $\langle X\rangle=\langle T\rangle=0$, is given by

$$
\begin{aligned}
\left.V\right|_{X=T=0}= & e^{K}\left[\lambda^{2}\left|S \tilde{S}-\mu^{2}\right|^{2}+v^{2}\left|S^{n} e^{a \Phi}+\tilde{S}^{n} e^{-a \Phi}\right|^{2}\right] \\
& +\frac{e^{2}}{2}\left(|\tilde{S}|^{2}-|S|^{2}+\frac{n}{a}\left(\Phi+\Phi^{*}\right)\right)^{2}
\end{aligned}
$$

with $K=\chi^{2}+|S|^{2}+|\tilde{S}|^{2}$. It is manifest that this effective potential is positive definite and its global minimum is given by the conditions

$$
\begin{aligned}
S \tilde{S}-\mu^{2} & =0, \\
S^{n} e^{a \Phi}+\tilde{S}^{n} e^{-a \Phi} & =0, \\
|\tilde{S}|^{2}-|S|^{2}+\frac{n}{a}\left(\Phi+\Phi^{*}\right) & =0 .
\end{aligned}
$$

These conditions lead to the global minimum for the fields, as

$$
\begin{gathered}
S_{\min }=\mu e^{i \beta}, \\
\tilde{S}_{\min }=\mu e^{-i \beta},
\end{gathered}
$$

$$
\begin{gathered}
\chi_{\min }=0 \\
\phi_{\min }=-\frac{\sqrt{2} n \beta}{a}+\frac{(2 m-1)}{\sqrt{2} a} \pi,
\end{gathered}
$$

where $m$ is an integer number.

However, when hybrid inflation ends and the shift symmetry is broken with $S \tilde{S}=\mu^{2}$, the imaginary part of $\Phi$ does not necessarily stay at the minimum, because before the breaking of the shift symmetry all the values of the imaginary part of $\Phi, \phi$, are equally distributed, thanks to the shift symmetry. Thus, the initial condition of $\phi$ is determined accidentally. The effective potential is given by

$$
\begin{aligned}
V_{\mathrm{eff}}= & e^{K} v^{2}\left|S_{\min }^{n} e^{a \Phi}+\tilde{S}_{\min }^{n} e^{-a \Phi}\right|^{2} \\
= & e^{\chi^{2}+2 \mu^{2}} \cdot v^{2} \mu^{2 n}\left[e^{\sqrt{2} \chi / M}+e^{-\sqrt{2} \chi / M}\right. \\
& \left.+2 \cos \left(2 n \beta+\frac{\sqrt{2} \phi}{M}\right)\right],
\end{aligned}
$$

with $M=1 / a$. Here, let us identify the inflaton and the Nambu-Goldstone (NG) boson correctly, which are given by

$$
\begin{aligned}
& \phi_{\mathrm{inf}}=\phi+\frac{n M}{\mu} \beta_{c}, \\
& \phi_{\mathrm{NG}}=\phi-\frac{n M}{\mu} \beta_{c},
\end{aligned}
$$

with $\beta_{c} \equiv \sqrt{2} \mu \beta$. Then, the covariant kinetic terms are given by

$$
\begin{aligned}
\frac{1}{2}\left(D_{\mu} \phi\right)^{2}+\frac{1}{2}\left(D_{\mu} \beta_{c}\right)^{2}= & \frac{1}{2}\left[\frac{1}{4}\left(1+\frac{\mu^{2}}{n^{2} M^{2}}\right)\left\{\left(\partial_{\mu} \phi_{\mathrm{inf}}\right)^{2}+\left(\partial_{\mu} \phi_{\mathrm{NG}}\right)^{2}\right\}+\left(1-\frac{\mu}{n M}\right) \partial_{\mu} \phi_{\mathrm{inf}} \partial^{\mu} \phi_{\mathrm{NG}}\right. \\
& \left.+\sqrt{2} n M A_{\mu}\left\{\left(1-\frac{\mu^{2}}{n^{2} M^{2}}\right) \partial^{\mu} \phi_{\mathrm{inf}}+\left(1+\frac{\mu^{2}}{n^{2} M^{2}}\right) \partial^{\mu} \phi_{\mathrm{NG}}\right\}+2\left(n^{2} M^{2}+\mu^{2}\right) A_{\mu} A^{\mu}\right],
\end{aligned}
$$

where

$$
D_{\mu} \phi \equiv \partial_{\mu} \phi+\sqrt{2} n M A_{\mu}, \quad D_{\mu} \beta_{c} \equiv \partial_{\mu} \beta_{c}-\sqrt{2} \mu A_{\mu},
$$

with $A_{\mu}$ being the gauge field. The NG boson $\phi_{\mathrm{NG}}$ is eaten by the gauge field, so the remaining kinetic terms in the unitary gauge become

$$
\begin{aligned}
\frac{1}{2} & {\left[\frac{1}{4}\left(1+\frac{\mu^{2}}{n^{2} M^{2}}\right)\left(\partial_{\mu} \phi_{\mathrm{inf}}\right)^{2}+\sqrt{2} n M\left(1-\frac{\mu^{2}}{n^{2} M^{2}}\right) A_{\mu} \partial^{\mu} \phi_{\mathrm{inf}}+2\left(n^{2} M^{2}+\mu^{2}\right) A_{\mu} A^{\mu}\right] } \\
& =\frac{1}{2} \frac{1}{1+\frac{n^{2} M^{2}}{\mu^{2}}}\left(\partial^{\mu} \phi_{\mathrm{inf}}\right)^{2}+\left(\mu^{2}+n^{2} M^{2}\right) \tilde{A}_{\mu} \tilde{A}^{\mu} \\
& =\frac{1}{2}\left(\partial^{\mu} \tilde{\phi}_{\mathrm{inf}}\right)^{2}+\left(\mu^{2}+n^{2} M^{2}\right) \tilde{A}_{\mu} \tilde{A}^{\mu}
\end{aligned}
$$


where

$$
\begin{gathered}
\tilde{A}_{\mu} \equiv A_{\mu}+\frac{n M-\frac{\mu^{2}}{n M}}{\sqrt{2}\left(\mu^{2}+n^{2} M^{2}\right)} \partial_{\mu} \phi_{\mathrm{inf}}, \\
\tilde{\phi}_{\mathrm{inf}} \equiv \sqrt{\frac{1}{1+\frac{n^{2} M^{2}}{\mu^{2}}}} \phi_{\mathrm{inf}} .
\end{gathered}
$$

Thus, the effective potential for the canonically normalized inflaton $\tilde{\phi}_{\text {inf }}$ is given by

$V_{\mathrm{eff}}\left(\tilde{\phi}_{\mathrm{inf}}\right)=2 e^{2 \mu^{2}} \cdot v^{2} \mu^{2 n} \cos \left(\frac{\sqrt{2}}{M} \sqrt{1+\frac{n^{2} M^{2}}{\mu^{2}}} \tilde{\phi}_{\mathrm{inf}}\right)$,

where the decay constant $f$ is given by

$$
f=\frac{M}{\sqrt{2}} \sqrt{\frac{1}{1+\frac{n^{2} M^{2}}{\mu^{2}}}} \rightarrow \frac{\mu}{\sqrt{2} n} \quad \text { for } n M \gg \mu .
$$

Thus, since $n$ is an integer number and larger than unity in this simple example, the decay constant $f$ cannot be superPlanckian scale as long as $\mu$ is sub-Planckian scale. So, inflation becomes hilltop type.

In order to reheat the Universe after inflation, we introduce the following superpotential:

$$
W_{R}=y S^{n} e^{a \Phi} N N,
$$

where $y$ is a (Yukawa) coupling constant and $N$ is the righthanded neutrino superfield. This superpotential with the canonical Kähler potential for $N$ is manifestly invariant under the shift symmetry Eqs. (12) and (15), and $N \rightarrow N$. Once $S$ acquires the nonzero VEV, this superpotential leads to a Yukawa coupling between the inflaton $\phi$ and the righthanded neutrino $\tilde{N}$. Therefore, the leptogenesis through the inflaton decay and the reheating of the Universe through the decay of the right-handed neutrino to the standard particles are possible by tuning the parameters adequately.

\section{CONCLUSIONS AND DISCUSSIONS}

In this paper, we constructed a concrete example of the dynamical breaking of the shift symmetry in SUGRA. By taking the exponential type of the superpotential for $\Phi$, which might appear through some nonperturbative effects, we first consider the superpotential invariant under the shift symmetry. Then, by arranging the grand unified theory
Higgs-like superpotential as well, the shift symmetry is dynamically broken. The inflaton has no dangerous exponential factor at the leading order in the scalar potential even after the shift symmetry breaking. Such an exponential factor is the main obstacle to realizing super-Planckian inflation in supergravity. Unfortunately, in our simple model, the potential obtained for the inflaton is a cosinetype potential rather than a power-law one, and it is difficult to realize a super-Planckian decay constant. One possible way to obtain the super-Planckian decay constant with $\mu$ being the sub-Planckian scale is to make $n$ smaller than unity. Of course, $n$ is an integer number and larger than unity in this simple example. However, for example, if we start from the higher order Kähler potential for $S(\tilde{S})$ like $|S|^{2 m}\left(|\tilde{S}|^{2 m}\right)$ instead of the canonical Kähler potential [with $S \tilde{S}$ replaced by $(S \tilde{S})^{m}$ in the superpotential $W_{B}$ at the same time], then such a model becomes equivalent to our simple model with the effective $n_{\text {eff }}=n / m$ by field redefining $S^{\prime} \equiv S^{m}\left(\tilde{S}^{\prime} \equiv \tilde{S}^{m}\right)$. Thus, if we take $n_{\text {eff }} \lesssim \mu / M_{p} \lesssim 1$, the decay constant $f \gtrsim M_{p}$, which may realize super-Planckian inflation like natural inflation. We leave a more realistic realization of natural and chaotic inflation as a future work.

We have restricted ourselves within the regime of EFT, where the fields have masses and energy densities below the cutoff in spite of the fact that the inflaton VEV could be large and above the Planck scale. We have also pointed out that it is possible to generate small inflaton couplings to matter in order to avoid some of the quantum corrections to the inflaton potential [11]. In this paper, we have explicitly assumed that all the fields are slow rolling initially in some patch of the Universe, such that the kinetic energy is indeed subdominant to be well within the regime of EFT.

\section{ACKNOWLEDGMENTS}

We thank Keisuke Harigaya, Masahiro Ibe, and Jun'ichi Yokoyama for helpful and instructive comments and discussions. A. M. is supported by the LancasterManchester-Sheffield Consortium for Fundamental Physics under STFC Grant No. ST/J000418/1. The work of T. N. is supported in part by the Special Postdoctoral Researcher Program at RIKEN. M. Y. is supported in part by the JSPS Grant-in-Aid for Scientific Research No. 25287054 and No. 2661006.

Note added.-Recently, Ref. [18] appeared, in which a similar breaking of shift symmetry is given, though the model is rather different and more complicated. We thank the authors of Ref. [18] for notifying us of that fact. 
[1] G. Hinshaw et al. (WMAP Collaboration), Astrophys. J. Suppl. Ser. 208, 19 (2013).

[2] P. A. R. Ade et al. (Planck Collaboration), arXiv:1303.5082.

[3] A. A. Starobinsky, Phys. Lett. 91B, 99 (1980); A. H. Guth, Phys. Rev. D 23, 347 (1981); K. Sato, Mon. Not. R. Astron. Soc. 195, 467 (1981).

[4] P. A. R. Ade et al. (BICEP2 Collaboration), Phys. Rev. Lett. 112, 241101 (2014).

[5] D. H. Lyth, Phys. Rev. Lett. 78, 1861 (1997).

[6] S. Choudhury and A. Mazumdar, Nucl. Phys. B882, 386 (2014); S. Hotchkiss, A. Mazumdar, and S. Nadathur, J. Cosmol. Astropart. Phys. 02 (2012) 008.

[7] S. Choudhury and A. Mazumdar, arXiv:1403.5549; arXiv: 1404.3398.

[8] A. R. Liddle, A. Mazumdar, and F. E. Schunck, Phys. Rev. D 58, 061301 (1998); E. J. Copeland, A. Mazumdar, and N. J. Nunes, Phys. Rev. D 60, 083506 (1999); A. Jokinen and A. Mazumdar, Phys. Lett. B 597, 222 (2004).

[9] P. Kanti and K. A. Olive, Phys. Lett. B 464, 192 (1999); Phys. Rev. D 60, 043502 (1999).

[10] A. D. Linde, Prog. Theor. Phys. Suppl. 163, 295 (2006).

[11] D. Chialva and A. Mazumdar, arXiv:1405.0513; A. Kehagias and A. Riotto, Phys. Rev. D 89, 101301 (2014).

[12] A. Mazumdar and J. Rocher, Phys. Rep. 497, 85 (2011).

[13] C. P. Burgess and M. Williams, arXiv:1404.2236.

[14] M. Kawasaki, M. Yamaguchi, and T. Yanagida, Phys. Rev. Lett. 85, 3572 (2000).
[15] M. Kawasaki, M. Yamaguchi, and T. Yanagida, Phys. Rev. D 63, 103514 (2001).

[16] K. Freese, J. A. Frieman, and A. V. Olinto, Phys. Rev. Lett. 65, 3233 (1990); F. C. Adams, J. R. Bond, K. Freese, J. A. Frieman, and A. V. Olinto, Phys. Rev. D 47, 426 (1993).

[17] K. Freese and W. H. Kinney, arXiv:1403.5277; M. Czerny, T. Higaki, and F. Takahashi, Phys. Lett. B 734, 167 (2014); K. Choi, H. Kim, and S. Yun, Phys. Rev. D 90, 023545 (2014); R. Kallosh, A. Linde, and B. Vercnocke, arXiv: 1404.6244; K.-Y. Choi and B. Kyae, Phys. Rev. D 90, 023536 (2014); T. Higaki, T. Kobayashi, O. Seto, and Y. Yamaguchi, arXiv:1405.0775; T. Higaki and F. Takahashi, arXiv:1404.6923; R. Kappl, S. Krippendorf, and H.P. Nilles, arXiv:1404.7127; C. Long, L. McAllister, and P. McGuirk, Phys. Rev. D 90, 023501 (2014); K. Kohri, C. S. Lim, and C.-M. Lin, arXiv:1405.0772 [J. Cosmol. Astropart. Phys. (to be published)].

[18] T. Li, Z. Li, and D. V. Nanopoulos, J. High Energy Phys. 07 (2014) 052.

[19] M. Yamaguchi, Classical Quantum Gravity 28, 103001 (2011).

[20] R. Kallosh, A. Linde, and T. Rube, Phys. Rev. D 83, 043507 (2011).

[21] D. Baumann and D. Green, J. High Energy Phys. 03 (2012) 001.

[22] G. R. Dvali, Q. Shafi, and R. K. Schaefer, Phys. Rev. Lett. 73, 1886 (1994).

[23] A. D. Linde and A. Riotto, Phys. Rev. D 56, R1841 (1997). 